\title{
Uso de la evidencia y significatividad histórica en la enseñanza de la Transición española mediante un entorno digital de aprendizaje
}

\section{Use of Evidence and Historical Significance when Teaching the Spanish Transition to Democracy by means of a Digital Learning Environment}

\author{
Diego Miguel-Revilla ${ }^{1}$ \\ dmigrev@sdcs.uva.es \\ Mercedes Calle-Carracedo \\ mcalle@sdcs.uva.es \\ María Sánchez-Agustí \\ almagosa@sdcs.uva.es \\ Universidad de Valladolid, España
}

\section{Resumen:}

INTRODUCCIÓN. Este estudio, enmarcado en el Proyecto HISREDUC, recoge los resultados obtenidos tras una intervención en la asignatura Geografía e Historia de cuarto curso de la ESO. MÉTODO. Se establece el objetivo de facilitar el desarrollo de dos dimensiones del pensamiento histórico (el uso de la evidencia y la percepción de la significatividad), mediante la aplicación de un entorno digital de aprendizaje durante siete sesiones, con un total de 53 estudiantes, y utilizando la enseñanza de la Transición española como marco curricular de trabajo. Mediante el uso de un análisis cualitativo, se examina el

\begin{abstract}
:
INTRODUCTION. This study, which is framed within the HISREDUC Project, presents the results obtained following an intervention with 15-year-old secondary education History students. METHOD. The aim of this paper is the development of two main concepts related to historical thinking (use of evidence and historical significance) through the use of a digital learning environment over seven sessions. 53 students participated and the curricular framework used was the Spanish transition to democracy. The level of progression related to both concepts is examined through a qualitative method which
\end{abstract}

1 Dirección para correspondencia (correspondence address):

Diego Miguel Revilla. Universidad de Valladolid. Departamento de Didáctica de las Ciencias Experimentales, Sociales y de la Matemática. Facultad de Educación y Trabajo Social. Paseo de Belén, 1. 47011 Valladolid (España). 
Uso de la evidencia y significatividad histórica en la enseñanza de la Transición española mediante un entorno digital de aprendizaje

Diego Miguel-Revilla, Mercedes Calle-Carracedo y María Sánchez-Agustí

grado de progresión mostrado en torno a ambas dimensiones, comparando el nivel de competencia presente antes y después de la aplicación del entorno digital. RESULTADOS. Los resultados permiten identificar patrones comunes ligados con las habilidades de los estudiantes, mostrando una evolución positiva en relación con el uso de la evidencia y con la comprensión de la significatividad histórica. DISCUSIÓN. Se concluye que la intervención didáctica utilizada ha reforzado y ayudado a hacer explícitas capacidades latentes, facilitando su aplicación en el análisis del pasado.

\section{Palabras clave:}

Pensamiento histórico; enseñanza de la historia; entornos digitales de aprendizaje; herramientas digitales; tecnología; fuentes históricas; historia reciente compared the perceived proficiency level before and after the use of the digital learning environment. RESULTS. The results allow to identify common patterns related to students' abilities, showing a positive progression related to the use of evidence and historical significance. DISCUSSION. It can be concluded that this intervention reinforced and helped made explicit latent capabilities, allowing their use in the analysis of the past.

\section{Key words:}

Historical thinking; history education; digital learning environments; digital tools; technology; historical sources; recent history

\section{Résumé:}

INTRODUCTION. Cette étude, dans le cadre du projet HISREDUC, présente les résultats obtenus après une intervention dans la matière $d^{\prime}$ histoire de quatrième année de I'ESO (15 ans). MÉTHODE. Avec l'objectif de développer deux dimensions de la pensée historique (I'utilisation de la preuve et la perception de signifiance). Cette intervention a été développée et appliquée à 53 étudiants à travers un environnement d'apprentissage numériques et en utilisant l'enseignement de transition espagnol comme cadre didactique. Grâce à une analyse qualitative, le degré de progression montré autour des deux dimensions est examiné, en comparant le niveau de la concurrence présente avant et après I'application de l'environnement numérique. RÉSULTATS. Les résultats permettent d'identifier des modèles communs liés aux capacités des élèves, montrant une tendance positive en ce qui concerne l'utilisation de la preuve et la compréhension de I'importance historique. DISCUSSION. En conclusion, l'intervention éducative utilisée a renforcé et contribué à faire des capacités latentes explicites, ce qui facilite son application dans l'analyse du passé.

\section{Mots clés:}

Pensée historique; enseignement de I'histoire; environnements numériques d'apprentissage; outils numériques; technologie; sources historiques; histoire récente.

Fecha de recepción: 22-06-2017

Fecha de aceptación: 29-03-2019

\section{Introducción}

La capacidad de razonar históricamente, un aspecto que Wineburg describió hace casi dos décadas como no natural y, por tanto, como algo 
que "va a contracorriente de cómo pensamos de forma habitual" (2001, p. 7), ha atraído la atención de los investigadores educativos, quienes han identificado su desarrollo como un pilar esencial de la formación histórica. El tratamiento de esta destreza, caracterizada por Lévesque como el "proceso intelectual a través del cual un individuo domina (y posteriormente apropia) los conceptos y el conocimiento de la Historia" (2008, p. 27), siempre con el objetivo de su aplicación crítica para la resolución de problemas, se convierte, por tanto, en fundamental a la hora de ir más allá de la enseñanza del conocimiento meramente factual, y comenzar a trabajar sobre el pasado de una forma más analítica y metódica.

Ante este reto, la introducción de las tecnologías en el aula se convierte en una oportunidad para estructurar un nuevo modelo de trabajo que facilite el desarrollo del pensamiento histórico, y de ahí que el Proyecto HISREDUC centre su atención en la utilización de fuentes históricas digitalizadas para la enseñanza de la Historia más reciente. Por supuesto, esto supone una transformación del proceso de enseñanza-aprendizaje, por lo que resulta de interés conocer no solo el grado de desarrollo de diversas capacidades relacionadas con la comprensión histórica en los alumnos, sino la forma en que la aplicación de herramientas digitales puede facilitar esta progresión.

\section{Marco teórico}

El énfasis en un nuevo tipo de orientación en la enseñanza tiene mucho que ver con los diferentes campos o dominios de conocimiento propios de la Historia, demarcados por VanSledright y Limón (2006) como sustantivos (tanto de primer como de segundo orden) y estratégicos. Si bien el conocimiento sustantivo de primer orden se encarga de responder a preguntas como quién, qué o cuándo, el de segundo orden atiende a conceptos más complejos, como la causalidad, las transformaciones o los conceptos de progreso y declive. El conocimiento estratégico, relacionado con aspectos metacognitivos y procedimentales, se relaciona, en cambio, con las prácticas propias de los historiadores, incluyendo el análisis de fuentes y su atención tanto a la perspectiva histórica de los protagonistas como al contexto del periodo analizado. En un sentido muy similar se sitúa la diferenciación establecida por Domínguez entre 
el conocimiento de y sobre la Historia, ya que mientras el primero trata sobre aquello que se sabe acerca del pasado, el segundo incluye "los conceptos, métodos y reglas" (2015, p. 44) propios de la investigación histórica.

El pensamiento o razonamiento histórico se relaciona con una interacción entre estos tipos de conocimiento, aunque de forma más directa con el conocimiento estratégico y, por tanto, con habilidades (análisis, juicio y empatía) y conceptos (evidencia, cambio y continuidad en el tiempo, causa y motivación, y anacronismo) fundamentales establecidos por primera vez, de forma sistemática, en el Schools Council History 13-16 Project en los años 70. Si bien la tradición británica, siguiendo el modelo de Shemilt y las investigaciones de Dickinson, Lee y Ashby, ha acabado concentrando su atención en conceptos básicos como el tiempo, la evidencia, el cambio, la empatía, las causas y los testimonios (Lee, 2005), es la escuela canadiense, encabezada por Seixas quien se ha centrado en establecer un modelo completo y coherente en las últimas tres décadas (Lévesque y Clark, 2018). Seixas, que inicialmente añadió a los marcos existentes aspectos relacionados con la idea de agencia histórica y los juicios morales, y que después apuntó la necesidad de atender a la significatividad histórica, ha refinado con el tiempo la selección de conceptos básicos, demarcando las seis principales dimensiones del pensamiento histórico: uso de la evidencia, significatividad histórica, continuidad y cambio, causa y consecuencia, perspectiva histórica y dimensión ética (Seixas y Morton, 2013). La presente investigación centra su atención específicamente en los dos primeros conceptos.

En el caso de la primera dimensión, el tratamiento de la evidencia histórica no se advierte como únicamente útil a la hora de transmitir las herramientas clave con las que analizar el pasado, sino también como apoyo para comprender cómo se construyen los relatos históricos a lo largo del tiempo. Después de todo, tal y como apunta VanSledright, el trabajo con fuentes puede entenderse como "discutiblemente, el sine qua non del pensamiento histórico" (2004, p. 231), ya que el énfasis en la contextualización y análisis crítico de los restos del pasado puede ayudar al alumnado a trabajar los procesos de inferencia, a entender la existencia de una pluralidad de visiones, a saber realizar las preguntas adecuadas y a evitar las dicotomías más simplistas en sus análisis.

La segunda dimensión analizada tiene que ver con la capacidad mostrada por los estudiantes para comprender la significatividad histórica de 
procesos y acontecimientos. Descrita como el criterio por el que ajustar los eventos del pasado a una Historia coherente, siempre en relación con el presente (Seixas, 2017), la idea de significatividad no solo se concibe como una percepción de la importancia, sino que se relaciona con las narrativas y testimonios históricos, otorgando valores de significatividad siempre en relación con otros eventos del pasado (Cercadillo, Chapman, y Lee, 2017).

Ahora bien, a pesar del hincapié puesto por los distintos investigadores en los procedimientos, habilidades o competencias, los modelos más amplios ligados con el pensamiento o el razonamiento histórico no pueden dejar de lado el conocimiento sustantivo de primer orden, siendo un reto su integración con los de segundo orden (Gómez, Ortuño, y Molina, 2014), atendiendo también a los elementos propios de la imaginación y la representación histórica (González, Pagès, y Santisteban, 2011), así como a la complementación de los aspectos más cognitivos con los relacionados con los intereses y funciones de la Historia (Chapman, 2014). Es ante esta oportunidad donde marcos conceptuales como el establecido por van Drie y van Boxtel (2018) pueden ser útiles, complementando las dimensiones clásicas propuestas por Seixas con elementos como la utilización de conceptos sustantivos o de una adecuada argumentación, lo que ha Ilevado a dar una mayor relevancia a las narrativas históricas (Rüsen, 2005; Sáiz-Serrano y López-Facal, 2015) o a las creencias epistemológicas sobre este campo de conocimiento (Stoel, van Drie, y van Boxtel, 2017).

El desafío es, por tanto, integrar todos estos aspectos citados con anterioridad, relacionados con la identificación, trabajo y evaluación en torno al pensamiento histórico, con la utilización de herramientas digitales para la enseñanza de la Historia, un campo Ileno de potencial (Haydn, 2011; Kee, 2014), pero también de dificultades prácticas que desde aquí tratan de despejarse con el objetivo de identificar y transformar la forma en la que se trabaja sobre el pasado en las aulas.

\section{Diseño de la investigación}

\section{Objetivos}

El objetivo general de este estudio es analizar el impacto del uso de una propuesta didáctica, integrada en un entorno digital de aprendizaje, en 
el desarrollo de conceptos clave del pensamiento histórico. Los objetivos específicos son dos: examinar, en primer lugar, el nivel de progresión mostrado por los participantes en relación con el tratamiento de la evidencia histórica y, en segundo lugar, respecto a la percepción de la significatividad histórica, estableciendo en ambos casos una comparativa para comprobar su grado de evolución tras la intervención.

\section{Metodología}

El presente estudio integra en su diseño elementos propios tanto de las investigaciones exploratorias o descriptivas (al buscar examinar las ideas y capacidades cognitivas de los estudiantes), como de las investigaciones aplicadas (al programar una intervención con la intención de provocar una transformación) (Cohen, Manion, y Morrison, 2005). Se trata de un tipo de investigación de carácter mixto, que combina una aproximación eminentemente cualitativa, adecuada para captar elementos dependientes del contexto e interpretaciones del alumnado (Stake, 2010), con un tratamiento cuantitativo de la información dentro de un diseño CUALcuan, en el que prima el primero de los componentes (Hernández-Sampieri, Fernández-Collado, y Baptista-Lucio, 2014). La valoración cualitativa de la información se ha efectuado mediante un examen centrado en los niveles de complejidad cognitiva en torno a competencias ligadas al pensamiento histórico, partiendo de categorías de análisis definidas a priori complementadas con el surgimiento de otras emergentes (Corbin y Strauss, 2015). Esta categorización ha permitido una comparativa descriptiva posterior de carácter cuantitativo para poner en relación los resultados obtenidos antes y después de la intervención.

\section{Participantes}

La investigación pudo contar como participantes con dos grupos de alumnos de un IES de la periferia de Valladolid matriculados en el cuarto curso de Educación Secundaria Obligatoria durante el curso 2015/2016. Pese a que un total de 53 estudiantes, con edades comprendidas entre los 15 y 16 años, trabajaron durante siete sesiones, por parejas, en torno a la Transición española, la muestra efectiva se ha reducido a 41 estudiantes (22 alumnos y 19 alumnas), ya que los distintos cuestionarios individuales no pudieron ser cumplimentados por la totalidad de los 
estudiantes debido a ausencias puntuales al principio o al final de la intervención.

\section{Características del entorno digital}

El entorno, de nueva creación, fue elaborado mediante la plataforma Moodle, un sistema de gestión del aprendizaje (LMS) de código abierto que facilita un elevado nivel de interacción y permite el seguimiento por parte de los docentes de la actividad realizada por sus alumnos, ofreciendo la capacidad de compartir unidades didácticas, actividades o recursos. El entorno se complementó con un repositorio de recursos históricos basado en Omeka, categorizando desde textos a elementos multimedia, y utilizando metadatos para facilitar la reutilización de los objetos de aprendizaje (Spector, Merrill, Elen, y Bishop, 2014).

La unidad utilizada, centrada en la Transición española, una temática de reconocida relevancia por la historiografía contemporánea, se estructuró en cinco sesiones prácticas (junto a dos adicionales de evaluación), cada una de ellas centrada en contenidos concretos, pero también en el desarrollo de habilidades específicas mediante el trabajo con actividades donde se integraron fuentes de diverso tipo, con las que los alumnos trabajaron directamente. Las sesiones no contemplaron únicamente temáticas tradicionales de contenido político, sino que se centraron en aspectos como la vida cotidiana, los cambios de mentalidad o la relación entre el pasado y la actualidad, siempre integrando herramientas participativas, desde el uso de foros a líneas temporales o recursos digitales interactivos.

\section{Instrumentos de análisis y categorización}

Con el objetivo de evaluar las ideas relacionadas con el uso de la evidencia y la percepción de la significatividad histórica, se diseñaron dos cuestionarios de respuesta abierta. Éstos fueron revisados de forma previa, sufriendo modificaciones a lo largo de su proceso de elaboración. El primero, con un total de cuatro preguntas abiertas, fue aplicado de forma anterior a la intervención, evaluando ideas y procesos cognitivos previos de los estudiantes. El segundo de los cuestionarios, con siete preguntas, y concebido como una versión ampliada del primero, se aplicó a posteriori para evaluar el desarrollo de las ideas y conceptos. Los 
instrumentos no se centraron en aspectos meramente factuales, usando la Transición española como marco para examinar aspectos ligados a las dos dimensiones seleccionadas y las diferentes categorías de análisis: contextualización y análisis de fuentes, cuestionamiento e interpretación, y pluralidad de visiones (en relación con el uso de la evidencia), e identificación de la relevancia, relación pasado-presente y variabilidad temporal (sobre la significatividad histórica).

Tabla 1

Niveles de progresión en el desarrollo del uso de la evidencia

\begin{tabular}{lll}
\hline Dimensión & $\begin{array}{l}\text { Nivel de } \\
\text { desarrollo }\end{array}$ & Categorías de análisis \\
\hline $\begin{array}{l}\text { Uso de la } \\
\text { evidencia }\end{array}$ & $\begin{array}{l}\text { Desarrollo } \\
\text { nulo }\end{array}$ & $\begin{array}{l}\text { Los recursos históricos ni se contextualizan ni anali- } \\
\text { zanplemente se nombran. }\end{array}$
\end{tabular}

El pasado se asume como dado, sin dar posibilidad a que sea interpretado.

No se percibe la posibilidad de que dos fuentes históricas puedan contener información contradictoria.

Desarrollo Los recursos se contextualizan y analizan parcialincipiente mente, transcribiendo frases literales sin intención crítica.

Parece existir la conciencia de que las fuentes se pueden analizar, pero no hay preguntas de investigación.

Se indica la existencia de contradicciones, sin un cuestionamiento de por qué. Validez generalizada.

Desarrollo Los recursos históricos se contextualizan en un lugar intermedio o tiempo concreto, sintetizando sus ideas.

Existe un cuestionamiento acerca de aspectos tratados en las fuentes, por lo que se presentan dudas. Se indican las contradicciones y se cuestiona la validez de los testimonios encontrados.

Desarrollo Los recursos se contextualizan adecuadamente, avanzado cuestionando críticamente las ideas proporcionadas. Se hacen explícitas preguntas relevantes de investigación sobre aspectos esenciales. Se advierte de la contradicción y contextualizan las diversas visiones. Se ligan evidencia y validez.

Con el objetivo de concretar el examen cualitativo, distintos niveles de progresión fueron establecidos para cada dimensión, distinguiendo entre un grado de desarrollo nulo, incipiente, intermedio y avanzado, 
según la competencia mostrada en relación a las categorías de análisis. Estas diferencias aparecen explicitadas en las Tablas 1 y 2, correspondientes a la utilización de la evidencia y a la percepción de la significatividad histórica, respectivamente, y donde se ha tenido en cuenta el modelo establecido por Seixas y Morton (2013). Las respuestas procedentes de los alumnos, codificadas mediante ATLAS.ti, sirvieron para poder asignar a cada estudiante un nivel de desarrollo, utilizando SPSS para establecer la comparativa descriptiva.

Tabla 2

Niveles de progresión en el desarrollo de la percepción de la significatividad histórica

\begin{tabular}{|c|c|c|}
\hline Dimensión & $\begin{array}{l}\text { Nivel de } \\
\text { desarrollo }\end{array}$ & Categorías de análisis \\
\hline \multirow[t]{4}{*}{$\begin{array}{l}\text { Significatividad } \\
\text { histórica }\end{array}$} & $\begin{array}{l}\text { Desarrollo } \\
\text { nulo }\end{array}$ & $\begin{array}{l}\text { La importancia del periodo no es percibida } \\
\text { por los alumnos, y no se identifican procesos o } \\
\text { acontecimientos de relevancia. } \\
\text { No se valora la repercusión de los aconteci- } \\
\text { mientos del pasado en la realidad actual. } \\
\text { No se diferencia la percepción o variación de } \\
\text { la importancia a lo largo del tiempo. }\end{array}$ \\
\hline & $\begin{array}{l}\text { Desarrollo } \\
\text { incipiente }\end{array}$ & $\begin{array}{l}\text { La importancia del periodo se asume automáti- } \\
\text { camente, identificando procesos poco relevan- } \\
\text { tes. } \\
\text { Se liga de forma superficial los procesos históri- } \\
\text { cos del pasado con el presente. } \\
\text { Se apunta que acontecimientos y procesos pue- } \\
\text { den percibirse de forma desigual en el tiempo. }\end{array}$ \\
\hline & $\begin{array}{l}\text { Desarrollo } \\
\text { intermedio }\end{array}$ & $\begin{array}{l}\text { La importancia del periodo es reconocida por } \\
\text { los alumnos, identificando procesos con clara } \\
\text { repercusión en la época. } \\
\text { Se reconoce la influencia del pasado en el } \\
\text { presente. } \\
\text { Se explicita que la percepción de los procesos } \\
\text { ha variado en el tiempo. }\end{array}$ \\
\hline & $\begin{array}{l}\text { Desarrollo } \\
\text { avanzado }\end{array}$ & $\begin{array}{l}\text { La importancia del periodo se justifica, identifi- } \\
\text { cando los procesos más relevantes. } \\
\text { Se argumenta de forma concreta cómo afecta } \\
\text { el pasado en la actualidad. } \\
\text { Se habla de la variabilidad temporal y social en } \\
\text { la percepción del pasado con ejemplos. }\end{array}$ \\
\hline
\end{tabular}




\section{Resultados y discusión}

\section{El trabajo con la evidencia histórica}

La comparativa realizada entre el grado de progresión mostrado por los participantes antes de la intervención y una vez finalizado el trabajo con el entorno digital de aprendizaje muestra un avance favorable en relación con el uso de la evidencia histórica. Tal y como se observa en la Tabla 3, una gran mayoría de los estudiantes fueron encuadrados en el nivel 'desarrollo incipiente' tras la realización del análisis previo, mostrando un cierto potencial, pero sin llegar a manifestar un dominio de las competencias analizadas. Tras la finalización de la intervención, y después de contrastar las respuestas obtenidas mediante el cuestionario final, es posible constatar que casi la mitad de los estudiantes consiguieron aumentar su destreza y progresar hacia los niveles intermedio y avanzado, aunque en el análisis final únicamente 22 de los 41 estudiantes queden incluidos en estos dos niveles.

Tabla 3

Niveles de desarrollo en torno al uso de la evidencia antes y después de la intervención

\begin{tabular}{llrrrr}
\hline & & A. prev. $(\mathrm{n}=41)$ & \multicolumn{2}{c}{ A. post. $(\mathrm{n}=41)$} \\
\hline \multirow{3}{*}{ Uso de la } & $\mathrm{n}$ & $\%$ & $\mathrm{n}$ & $\%$ \\
evidencia & Desarrollo nulo & 2 & 4.9 & 0 & --- \\
& Desarrollo incipiente & 36 & 87.8 & 19 & 46.3 \\
& Desarrollo intermedio & 3 & 7.3 & 15 & 36.6 \\
& Desarrollo avanzado & 0 & --- & 7 & 17.1 \\
\hline
\end{tabular}

Nota. A. prev. = Análisis previo; A. post. = Análisis posterior.

Para el análisis en detalle del trabajo con la evidencia, se presta especial atención a tres categorías fundamentales: la contextualización y análisis de las fuentes, el cuestionamiento e interpretación de las mismas, y la valoración acerca de la pluralidad de visiones en la Historia.

Así, en relación con la capacidad de contextualizar y analizar críticamente la evidencia, los estudiantes muestran, en sus respuestas al cuestionario inicial, pautas comunes. Aunque en muy pocas ocasiones se obvian completamente las fuentes, sí que es posible detectar una tendencia generalizada a aludir a la evidencia con el único objetivo de describirla, de citarla de forma literal o meramente de transcribirla sin intención 
crítica. Concretamente, en el análisis de un texto periodístico del año 2012, en el que se vierten las opiniones de varios personajes públicos en torno a la muerte de Santiago Carrillo, los alumnos tienden a utilizar las mismas palabras o términos que éstos encuentran en la fuente usada. De esta manera, se alude constantemente al "papel imprescindible" del fallecido, describiéndole frecuentemente como "personaje clave" o como "referente", algo que no es más que un mero reflejo de la opinión expresada, con los mismos términos, en el propio texto.

Las respuestas a los cuestionarios finales aplicados tras la intervención, en la que los alumnos trabajaron acerca de la contextualización de la evidencia histórica, reflejan intentos algo más sofisticados, aunque limitados, de producir una síntesis capaz de situar los textos en sus contextos, otorgándoles cierto propósito. Es en este tipo de análisis cuando aparece el interés por el propio texto como objeto de examen, por su creador, o incluso, en alguna ocasión especial, por la intención de su uso. Como apunta un estudiante, todo "depende del punto de vista y la ideología de quien hable" [12.A, masc.], aspecto que lleva a algunos alumnos a identificar las preferencias ideológicas de los protagonistas o de los propios medios, como el caso de una alumna, para la que el análisis de los testimonios proporciona "una idea del tipo de ideales del $A B C$ y La Vanguardia [periódicos usados], [demostrando] que no hay una sola opinión sobre los hechos y que dependiendo de la fuente puedes encontrar una u otra" [53.B, fem.].

A pesar de todo, y con este último ejemplo como única excepción, la ausencia general de interés por los medios en los que los artículos fueron publicados (ni por sus autores, a pesar de estar identificados) indica una tendencia a no relacionar aspectos relevantes propios del contexto. De igual manera, los elementos puramente cronológicos no suelen hacerse explícitos por los participantes en sus análisis, y, aunque estos sí son capaces de distinguir entre el periodo en el que, por ejemplo, un documento fue escrito y la época a la que este se refiere, no valoran la importancia de este factor a la hora de realizar sus argumentaciones.

Por otro lado, en relación con el cuestionamiento e interpretación de la evidencia, la atención se centra específicamente en la capacidad de los estudiantes para concebir las fuentes como elementos analizables. La realización de preguntas de indagación desde una perspectiva crítica se convierte en un elemento a valorar, al encontrarse un contraste antes de 
la intervención y con posterioridad. En el primer caso, las descripciones literales de los textos detectadas en las respuestas Ilevan aparejada una concepción de éstos como cerrados, y por tanto, una ausencia completa de interrogantes, al asumir como incuestionables las premisas de las fuentes. De ahí que la forma de analizar la evidencia histórica se parezca más a una descripción de la realidad que a una interpretación, aunque sí que puedan encontrarse, en ocasiones, expresiones como "el texto nos da una imagen de la Transición, interpretándola como..." [11.A.pre, m.], o "[el artículo] nos presenta a..." [43.B.pre, f.], donde se percibe un matiz mucho más crítico.

El énfasis otorgado a las preguntas de investigación en ciertas actividades diseñadas para el entorno digital, centrándose de forma muy explícita en el qué, el cuándo, el quién o el cómo de los procesos históricos, ha facilitado una evolución detectable en la evaluación posterior. Junto a las respuestas menos críticas y literales, un número significativo de estudiantes (19 en total) incluyeron, con mayor o menor grado de espontaneidad, preguntas acerca de aspectos sobre los que les gustaría saber más sobre este proceso ("¿qué porcentaje de la población vivió bien el periodo [franquista]?" [36.A, f.]; “¿Cuánta gente pensó que su manera de intervenir en la política de España fue la correcta?" [8.A, f.]). El hecho de que un alumno indicara que un "historiador tiene otra forma de pensar a la de los políticos. [El texto] me sugiere que cuál de las dos será verdad" [13.A, m.], manifiesta que en casos como este existe una reflexión acerca de la propia naturaleza del relato.

El tercer aspecto sobre el que se hizo hincapié durante el trabajo en el aula fue la pluralidad de visiones, incluyendo la existencia de variadas interpretaciones y de informaciones o testimonios contrapuestos en torno a los acontecimientos del pasado. Preguntados por la forma en la que afrontar investigaciones históricas con múltiples perspectivas, las respuestas obtenidas mediante la aplicación del cuestionario inicial muestran una tendencia acrítica muy generalizada de entender como válidos todos los puntos de vista ("todos los testimonios tendrían que ser igual de válidos porque cada uno puede tener su propia opinión y hay que respetarlo" [44.B.pre, f.]; "cada persona tiene su punto de vista" [30.B.pre, f.]). Ahora bien, incluso antes del trabajo con el entorno, se detecta la capacidad de presentar opiniones con más matices. Si una alumna afirma que ella "intentaría sacar la información más neutral" [22.A.pre, f.] en sus investigaciones, un compañero advierte de que él 
"compararía la información con otras fuentes para saber cuál es el testimonio más correcto" [40.B.pre, m.,].

De forma práctica, las respuestas obtenidas, una vez acabada la intervención, tras la comparativa de distintos textos con apreciaciones contrapuestas en torno al legado de Santiago Carrillo, indican aproximaciones diversas. Aunque es posible encontrar con frecuencia valoraciones meramente descriptivas frente a información contrapuesta "había gente a favor y en contra de su política" [2.A, m.]; "sobre Santiago Carrillo hay muy diversas opiniones" [21.A, m.]), por lo general se detecta un intento de dar sentido a esta contradicción, pese a que en algunas ocasiones pueda detectarse un cierto nivel de confusión ("ipor qué Carrillo es tan bueno y a la vez tan malo?" [38.B, f.]). Por otro lado, ciertos estudiantes tratan de situarse en una posición más crítica, mostrando curiosidad por otros objetos de análisis, como la opinión de la sociedad del momento: "puedo obtener información y opiniones positivas y negativas que me sugieren preguntas como: ¿y qué pensaba la gente de a pie sobre este personaje?" [1.A, m.].

En el trasfondo de estos razonamientos se encuentran visiones tácitas acerca de los hechos, la objetividad o los conceptos de 'verdad' e 'interpretación', relacionados con las creencias epistémicas sobre la disciplina. De ahí que sea común encontrar, tras la intervención, reflexiones ("me surge la duda de cuál de los dos [testimonios] es el que más se acerca a la verdad" [51.B, m.]; "también te genera preguntas, como ¿cuál de los dos testimonios es más [válido (tachado por el alumno)] verdadero?" [15.A, $\mathrm{m}]$.$) donde se explicita una preferencia por dejar de lado los aspectos$ más subjetivos siempre que sea posible ("tomaría la [opinión] que considero más inteligente y daría información de manera objetiva" [3.A, m.]), y donde se introduce el concepto de validez basado en la evidencia.

\section{La percepción de la significatividad histórica}

Ante el examen de las respuestas proporcionadas, y atendiendo a la categorización establecida en niveles de progresión (Tabla 4), es posible advertir un avance positivo en la percepción de la significatividad histórica. Si bien, una gran mayoría de los alumnos comenzaron situados en los niveles de desarrollo nulo o incipiente, con posterioridad a la intervención es posible observar una mejora, pudiéndose categorizar a un total de 25 estudiantes en el nivel intermedio o en el avanzado. 
Uso de la evidencia y significatividad histórica en la enseñanza de la Transición española mediante un entorno digital de aprendizaje

Diego Miguel-Revilla, Mercedes Calle-Carracedo y María Sánchez-Agustí

Tabla 4

Niveles de desarrollo de la percepción de la significatividad histórica antes y después de la intervención

\begin{tabular}{llrrrc}
\hline & & A. prev. $(\mathrm{n}=41)$ & A. post. $(\mathrm{n}=41)$ \\
\hline \multirow{3}{*}{ Significatividad } & $\mathrm{n}$ & $\%$ & $\mathrm{n}$ & $\%$ \\
histórica & Desarrollo nulo & 4 & 9.8 & 0 & --- \\
& Desarrollo incipiente & 32 & 78.0 & 16 & 39.0 \\
& Desarrollo intermedio & 4 & 9.8 & 18 & 43.9 \\
& Desarrollo avanzado & 1 & 2.4 & 7 & 17.1 \\
\hline
\end{tabular}

Nota. A. prev. = Análisis previo; A. post. = Análisis posterior.

En esta ocasión se presta atención a tres categorías: la identificación de la relevancia del periodo analizado, el reconocimiento de la relación entre el pasado y el presente, y la variabilidad de la significatividad percibida a lo largo del tiempo.

En primer lugar, se atiende a la identificación de la relevancia de aquellos procesos históricos que provocaron transformaciones significativas o que influyeron de forma importante en la sociedad del momento. En relación con la Transición, las respuestas que se obtuvieron antes del trabajo de aula muestran una tendencia a denominar este proceso como "importante", "muy importante" o "cambio transcendental" de forma automática, sin mayor preocupación por entender qué hace de este periodo algo tan particular. La repetición mecánica de estas ideas no argumentadas no supone una reflexión de fondo, pero refleja los conocimientos previos, adquiridos, probablemente, por la presencia de este periodo en los medios y en el debate político actual.

En las respuestas obtenidas tras la intervención se aprecia, en cambio, una relación argumentada del periodo con aspectos como la recuperación de las libertades "la Transición es una época muy importante en nuestro país. Gracias a ella, España es un país libre en la que todas las personas somos 'iguales' y tenemos los mismos derechos" [24.A, m.]) o la transformación de la sociedad ("fue un proceso importante porque marcó un cambio de etapa mejor para la mayoría de los españoles" [52.C, f.]), pero también con la manera en que este cambio se produjo ("fue un proceso de gran importancia ya que sin la necesidad de una guerra civil se llevaron a cabo pactos entre partidos" [49.C, f.]). Estos argumentos guardan relación estrecha con los obtenidos en otra investigación realizada en Chile, un país con un proceso semejante al español (Sánchez-Agustí, Vásquez-Lara, y Vásquez-Leyton, 2016). 
Analizando con detalle los acontecimientos indicados por los estudiantes como más significativos, nombrados casi únicamente en las respuestas obtenidas una vez acabada la intervención, es relevante observar que la muerte de Franco es citada por una mayoría de los alumnos (17 ocasiones), seguida por la elaboración de la Constitución de 1978 (11 menciones), y la legalización de los partidos políticos y la celebración de elecciones (en 9 casos). Por detrás aparecen acontecimientos clave como la aprobación de la Ley de Amnistía, la coronación de Juan Carlos I o el nombramiento de Adolfo Suárez como Presidente del Gobierno.

Por supuesto, la idea de significatividad se relaciona también con el reconocimiento de la relación entre el pasado y el presente, incluyendo su influencia, efectos y repercusiones. En relación con este aspecto, las respuestas encontradas antes de la intervención son, por lo general, poco matizadas, no ligándose el pasado y el presente de forma explícita, o en todo caso, únicamente centrándose en aquellos elementos más visibles ("seguimos en una democracia" [9.A.pre, m.]; "gracias a ella [la Transición] mantenemos nuestro actual sistema político vigente" [23.A.pre, $\mathrm{m}]$.$) . Por supuesto, también se encuentran respuestas en las que las trans-$ formaciones no se reducen a los aspectos políticos ("la sociedad, economía, política y cultura actual se debe (sic) a todo aquello que a lo largo de la historia se ha aprendido, tras observar nuestros propios errores" [18.A.pre, m.]), indicando un tipo de análisis más complejo.

Entre las respuestas obtenidas en cuestionario final destaca un uso de argumentaciones algo más sutiles. Este es el caso de la explicación de un mismo alumno acerca de los elementos que han perdurado con el paso del tiempo ("la creación y evolución de unos partidos políticos [...], cada uno con su ideología, que siguen vigentes en la actualidad (PSOE), que han evolucionado ( $A P \rightarrow P P$ ) o que incluso han desaparecido"), pero también acerca de la influencia del periodo en el presente, donde, en su opinión, "actuales políticos y partidos imitan elementos de la Transición" [11.A, m.].

En tercer lugar, se analiza la variabilidad temporal de la significatividad acerca de los procesos históricos. Aunque los alumnos tienen más facilidad para comprender que los acontecimientos no siempre son percibidos de la misma forma por parte de todos los miembros de la sociedad, reconocer que el paso del tiempo puede provocar cambios en la forma de observar el pasado es algo más complejo. Las respuestas obtenidas antes de la intervención muestran que son pocos los estudiantes 
que niegan esta variación, aunque suelen relacionarla con intereses personales, ya sean económicos o políticos, como hace un alumno quien cree que la visión no será la misma con el tiempo "porque a unos los habrán (sic) afectado de distinta forma, a bien o a mal" [23.A.pre, m.], o como indica una compañera, para quien "no todas las personas vieron el cambio con los mismos ojos, ya que a los grandes cargos de la España Franquista acabaron perjudicando (sic) y su descendencia mantendrá esta ideología" [49.B.pre, f.].

En el caso de los resultados obtenidos tras la intervención, una gran mayoría de los participantes advierten de esta variabilidad temporal, aunque con distintos énfasis. Una de las alumnas, centrando su argumentación en los condicionamientos del pasado, hace referencia a que "la Transición ahora se ve con otros ojos con los que se veía antes porque antes tenían miedo a lo que pudiese pasar, y ahora la mayoría de las personas pensamos que ha sido algo bueno para tener libertades, entre otras cosas" [44.C, f.]. Este razonamiento, compartido por una de sus compañeras de grupo ("actualmente se verá diferente ya que la gente ya no tiene miedo a que una simple idea pueda conducir a una guerra civil" [49.C, f.]), marca como explícita la idea de que los cambios en la sociedad provocados por el paso del tiempo transforman cómo se ve un periodo, algo que se percibe también en las respuestas que no solo inciden en los condicionantes, sino también en los aspectos más políticos ("la valoración ha cambiado: muchos de los partidos actuales quieren cambiar algunas partes de la Constitución, ya que se escribió en una época distinta a la de ahora, y muchas cosas han cambiado" [51.C, m.]).

\section{Conclusiones}

El trabajo en torno al desarrollo de las diferentes dimensiones del pensamiento histórico es, debido a sus características, un proceso gradual que requiere de tiempo y que, a la vez, se ve condicionado por las transformaciones cognitivas de los estudiantes, influenciadas por la edad, la experiencia y el contexto socio-cultural (Carretero y Bermúdez, 2012). Ante la dificultad de establecer comparaciones absolutas, para esta investigación se ha optado por definir una serie de niveles de desarrollo en torno al uso de la evidencia y la percepción de la significatividad históri$\mathrm{ca}$, adaptados a las capacidades de los alumnos de Secundaria. 
Estos niveles de progresión, que oscilan entre el desarrollo nulo y el avanzado, no pretenden abarcar todas las posibilidades a la hora de trabajar sobre el pasado, sino que pretenden servir como demarcadores de los grados de dominio de conceptos de segundo orden en este curso de Educación Secundaria. Es en este marco donde debería entenderse la evolución advertida en los participantes, fruto además de la explicitación de unas posiciones que podían detectarse ya como latentes en muchos de los alumnos, y que, gracias al trabajo mediante el entorno digital de aprendizaje, se pudieron aplicar de forma práctica al análisis crítico del periodo estudiado. Es decir, los estudiantes no desarrollaron su comprensión de estos conceptos de segundo orden ex nihilo, sino que se valieron del trabajo realizado en las diversas sesiones para aplicar su capacidad de razonamiento, ya existente de forma más o menos efectiva, al campo del aprendizaje de la Historia.

Los alumnos mostraron un mayor dominio de las distintas competencias relacionadas con ambas variables en aquellas respuestas obtenidas tras la intervención. Atendiendo al primer objetivo específico, centrado en examinar el uso de la evidencia, las tendencias detectadas, de forma inicial, a expresar ideas o afirmaciones encontradas en las fuentes históricas de una forma literal y no crítica, fueron mitigadas por la insistencia en la contextualización durante el trabajo con la propuesta diseñada para la intervención. Esto es algo también detectable en relación con el cuestionamiento de las fuentes mediante preguntas de investigación, elemento clave no solo en el modelo de Seixas, sino también en el de razonamiento histórico elaborado por van Drie y van Boxtel (2018). De igual forma, la multiplicidad de actividades integradas en el entorno digital centradas en el trabajo contrafactual también parece haber facilitado un mayor cuestionamiento al respecto de conceptos tan importantes como la evidencia o la validez de testimonios.

En relación con el trabajo en torno a la significatividad histórica, foco del segundo objetivo específico, también es posible advertir una mayor matización posterior a la intervención a la hora de reconocer la relevancia de los procesos históricos, aunque este reconocimiento tenga que ver, en parte, con la obtención de un mayor conocimiento acerca del periodo durante el trabajo en el aula. Por otro lado, el hecho de que el diseño de la propuesta aplicada pusiera su foco en la relación tan marcada entre el pasado y el presente, con muchos ejemplos específicos, parece haber facilitado una comprensión por parte de los participantes acerca de 
las repercusiones que todavía perviven de los acontecimientos clave del pasado, así como de las transformaciones en las visiones y valoraciones con el paso del tiempo, aspectos propios de una visión más compleja de la significatividad (Cercadillo et al., 2017).

Respecto al propio entorno digital y su potencial utilidad a la hora de trabajar sobre los conceptos básicos relacionados con el pensamiento histórico, tal y como aparece reflejado en el objetivo general, la experiencia puede calificarse como positiva. La transformación relativa al modelo didáctico, llevada a cabo en clase, facilitó una potenciación de la autonomía de los alumnos, lo que les permitió a abordar la materia desde una perspectiva mucho más abierta a través una indagación más exhaustiva de fuentes históricas y otros recursos multimedia relacionados con la época de la Transición. Precisamente, el acercamiento a un tipo de trabajo algo más similar al que llevan a cabo los historiadores (Wineburg, Martin, y Monte-Sano, 2013), por supuesto, con sus limitaciones temporales, conceptuales y de método, es uno de los factores que, como es posible concluir, ha favorecido una mejor comprensión de los procedimientos y habilidades necesarias para un examen más profundo y más certero del pasado.

En todo caso, y pese a la necesidad de seguir transformando de forma más sofisticada la manera en la que se enfoca el estudio del pasado en las aulas, la complementación de los conocimientos puramente sustantivos de primer orden (y por tanto, de lo que sabemos de la Historia) con los de segundo orden y procedimentales (incluyendo aspectos sobre la propia Historia), se antoja una necesidad, sobre todo si lo que se busca es formar a estudiantes críticos y capaces de pensar de una manera más compleja y con más matices sobre todo lo que les rodea.

\section{Referencias}

Carretero, M., y Bermúdez, Á. (2012). Constructing Histories. En J. Valsiner (Ed.), The Oxford Handbook of Culture and Psychology (pp. 625-648). New York: Oxford University Press.

Cercadillo, L., Chapman, A., y Lee, P. (2017). Organizing the Past: Historical Accounts, Significance and Unknown Ontologies. En M. Carretero, S. Berger, y M. Grever (Eds.), Palgrave Handbook of Research in Historical Culture and Education (pp. 553-572). London: Palgrave.

Chapman, A. (2014). «But it Might Not Just Be Their Political Views»: Using Jörn Rüsen's 
Uso de la evidencia y significatividad histórica en la enseñanza de la Transición española mediante un entorno digital de aprendizaje Diego Miguel-Revilla, Mercedes Calle-Carracedo y María Sánchez-Agustí

«Disciplinary Matrix» to Develop Understandings of Historical Interpretation. Cadernos de Pesquisa, 9(21), 67-85.

Cohen, L., Manion, L., y Morrison, K. (2005). Research Methods in Education (5. ${ }^{\mathrm{a}} \mathrm{ed}$. .). London: Routledge.

Corbin, J., y Strauss, A. (2015). Basics of Qualitative Research: Techniques and Procedures for Developing Grounded Theory. Thousand Oaks: SAGE Publications.

Domínguez, J. (2015). Pensamiento histórico y evaluación de competencias. Barcelona: Graó.

Gómez, C. J., Ortuño, J., y Molina, S. (2014). Aprender a pensar históricamente. Retos para la historia en el siglo XXI. Tempo e Argumento, 6(11), 5-27. doi:10.5965/2175180306112014005

González, N., Pagès, J., y Santisteban, A. (2011). ¿Cómo evaluar el pensamiento histórico del alumnado? En P. Miralles, S. Molina, y A. Santisteban (Eds.), La evaluación en el proceso de enseñanza y aprendizaje de las Ciencias Sociales (pp. 221-232). Murcia: Universidad de Murcia y AUPDCS.

Haydn, T. (2011). History Teaching and ICT. En I. Davies (Ed.), Debates in History Teaching (pp. 236-248). London: Routledge.

Hernández-Sampieri, R., Fernández-Collado, C., y Baptista-Lucio, P. (2014). Metodología de la investigación (6. ${ }^{\mathrm{a}}$ ed.). México: McGraw-Hill.

Kee, K.B. (Ed.). (2014). Pastplay: Teaching and Learning History with Technology. Ann Arbor: University of Michigan Press. doi:10.3998/dh.12544152.0001.001

Lee, P. (2005). Putting Principles into Practice: Understanding History. En M.S. Donovan y J.D. Bransford (Eds.), How Students Learn: History in the Classroom (pp. 31-78). Washington, D.C.: The National Academies Press.

Lévesque, S. (2008). Thinking Historically: Educating Students for the Twenty-First Century. Toronto: University of Toronto Press.

Lévesque, S., y Clark, P. (2018). Historical Thinking: Definitions and Educational Applications. En S. Metzger y L.M. Harris (Eds.), The Wiley International Handbook of History Teaching and Learning (pp. 119-148). Malden: Wiley.

Rüsen, J. (2005). History: Narration, Interpretation, Orientation. New York: Berghahn Books.

Sáiz-Serrano, J., y López-Facal, R. (2015). Competencias y narrativas históricas: el pensamiento histórico de estudiantes y futuros profesores españoles de educación secundaria. Revista de Estudios Sociales, (52), 87-101. doi:10.7440/res52.2015.06

Sánchez-Agustí, M., Vásquez Lara, N., y Vásquez Leyton, G. (2016). Understanding The Processes Of Transition From Dictatorship To Democracy. A Survey Among Secondary Schools Students In Chile. International Journal of Historical Learning Teaching and Research, 13(2), 23-34.

Seixas, P. (2017). A Model of Historical Thinking. Educational Philosophy and Theory, 49(6), 593-605. doi:10.1080/00131857.2015.1101363

Seixas, P., y Morton, T. (2013). The Big Six Historical Thinking Concepts. Toronto: Nelson. Spector, J.M., Merrill, M.D., Elen, J., y Bishop, M.J. (Eds.). (2014). Technology-Based Instructional Design: Evolution and Major Trends. En Handbook of Research on Educational Communications and Technology (4. ${ }^{\mathrm{a}}$ ed., pp. 661-671). London: Springer. 
Uso de la evidencia y significatividad histórica en la enseñanza de la Transición española mediante un entorno digital de aprendizaje

Diego Miguel-Revilla, Mercedes Calle-Carracedo y María Sánchez-Agustí

Stake, R.E. (2010). Qualitative Research. Studying How Things Work. New York: The Guilford Press.

Stoel, G.L., van Drie, J., y van Boxtel, C. (2017). The Effects of Explicit Teaching of Strategies, Second-Order Concepts, and Epistemological Underpinnings on Students' Ability to Reason Causally in History. Journal of Educational Psychology, 109(3), 321-337. doi:10.1037/edu0000143

van Boxtel, C., y van Drie, J. (2018). Historical Reasoning: Conceptualizations and Educational Applications. En S. Alan Metzger y L.M. Harris (Eds.), The Wiley International Handbook of History Teaching and Learning (pp. 149-176). New York: Wiley-Blackwell.

VanSledright, B.A. (2004). What Does It Mean to Think Historically... and How Do you Teach It? Social Education, 68(3), 230-233.

VanSledright, B.A., y Limón, M. (2006). Learning and Teaching Social Studies: A Review of Cognitive Research in History and Geography. En P.A. Alexander y P.H. Winne (Eds.), Handbook of Educational Psychology (2. ${ }^{\text {a }}$ ed., pp. 545-570). New York: Routledge.

Wineburg, S. (2001). Historical Thinking and Other Unnatural Acts: Charting the Future of Teaching the Past. Philadelphia: Temple University Press.

Wineburg, S., Martin, D., y Monte-Sano, C. (2013). Reading Like a Historian. Teaching Literacy in Middle \& High School History Classrooms. New York: Teachers College Press.

\section{Financiación}

Esta investigación es parte del Proyecto HISREDUC ("La Historia reciente en la educación. Diseño y evaluación de entornos digitales de aprendizaje en la enseñanza secundaria de España y Chile", con referencia EDU2013-43782-P), financiado por el Ministerio de Economía, Industria y Competitividad (MINECO) del Gobierno de España. 